\title{
Journal policy on publishing studies with negative results
}

\author{
Martin CS Wong ${ }^{1,2}$ *, MD, MPH, Junjie Huang ${ }^{2}$, MD, MSc, David Weller ${ }^{3}$, MD, PhD, Roger Jones ${ }^{4}$, FRCP, FRCGP \\ ${ }^{1}$ Editor-in-Chief, Hong Kong Medical Journal \\ 2 Jockey Club School of Public Health and Primary Care, Faculty of Medicine, The Chinese University of Hong Kong \\ ${ }^{3}$ Centre for Population Health Science, The University of Edinburgh, Edinburgh, United Kingdom \\ ${ }^{4}$ School of Population Health and Environmental Science, King's College London, London, United Kingdom \\ *Corresponding author: wong_martin@cuhk.edu.hk
}

Hong Kong Med J 2019;25:424-6

https://doi.org/10.12809/hkmj195090

Contrast-enhanced computed tomography plays an important role in emergency and intensive care units. Contrast media is one of the most commonly used agents. Its administration may potentially lead to acute kidney injury (AKI). ${ }^{1}$ However, it remains unknown whether intravenous contrast media may increase the risk of AKI in patients with sepsis. ${ }^{2}$ Sepsis is a major cause of admission to intensive care settings and hospital death, and is also a risk factor for AKI. ${ }^{3,4}$ Therefore, it is important to study the association between contrast media and the incidence of AKI in patients with sepsis.

In this issue of the Hong Kong Medical Journal, Hsu et $\mathrm{al}^{5}$ conducted a study on patients with sepsis who received computed tomography scans with or without contrast media at a tertiary referral centre. The results showed no difference between the two groups in the incidence of AKI, emergent dialysis, mortality, and hospital stay. They concluded that intravenous contrast administration in computed tomographic scans was not associated with increased risk of AKI in patients with sepsis. These findings justified the practice of administering contrast to patients with sepsis in emergency and intensive care settings. As was mentioned by the authors, there were limitations in the study. The study was conducted in only a single site, limiting its generalisability to other populations in different hospital settings. Secondly, a causal relationship could not be established as the analysis was performed by reviewing electronic health records. There may have been selection bias as the control group consisted of patients with older age, higher blood pressure, and poorer renal function. Missing data on lactate and initial serum creatinine had also decreased the statistical power to detect an association. Despite the application of propensity score matching other residual confounders may exist.

Although the above study reported negative results, the Journal accepted this important original study due to its significant clinical implications. Studies that have included negative or inconclusive results, ${ }^{6,7}$ or those that are mostly descriptive in nature ${ }^{8,9}$ have been accepted by the Journal owing to their value and interest to readers in clinical practice or healthcare services. Studies with negative results are usually regarded less favourably in the scientific literature. ${ }^{10}$ Papers with statistically significant positive results are estimated to be 3 times more likely to be published than studies with negative results. ${ }^{11}$ In the past years, the proportion of studies with negative results published in scientific literature in most disciplines had been decreasing. ${ }^{12}$ This will bring forth some important issues, including publications bias.

\section{Consequences of not publishing negative results}

Publication bias is introduced when the results of a study influence the decision on whether to disseminate them. Publishing only statistically significant findings influences the balance of evaluation and results in potential bias. ${ }^{13}$ Investigation on publication bias is a key topic in systematic review and meta-analysis. For instance, the funnel plot is a useful tool to test the existence of publication bias in meta-analysis. ${ }^{14}$ Since there is a preference for publishing studies with positive results, the overall scientific literature contains many more Type I errors (false positive) than Type II errors (false negative). ${ }^{15}$ This is harmful as the Type I errors may mislead researchers, physicians, and policymakers on evaluating the benefits of an intervention. Awareness of publication bias may deter investigators from submitting negative trials in the first place.

The preference for positive results also contributes to the phenomenon of hypothesising after the results are known (also known as HARKing). ${ }^{16}$ This happens when researchers review their study results and change their hypotheses without acknowledgement of this process. This commonly observed form of data misinterpretation may be caused by increasing competition in science among researchers. To identify positive results, researchers tend to focus on statistically significant positive results rather than negative ones. More seriously, there are some reports on scientific misconduct of falsifying the data among researchers. ${ }^{17}$ 
Not publishing studies with negative results can waste the time, money, and resources of not only those researchers but also of other researchers exploring similar lines of research. Consequently, this vicious circle results in personal discouragement and a significant waste of research resources that could have been allocated to other areas. Although finding that a treatment is ineffective may not be as interesting as positive results, it is a valuable result and worth sharing with the community, provided the study is properly designed and conducted. By doing this, it is unnecessary for other researchers to duplicate and they are less prone to study on the same research question. When healthcare resources are limited, it is important for policymakers to know which interventions are effective and which are not.

Additionally, it may indirectly increase the health risk for patients who are involved in a similar clinical trial using ineffective treatment. Publishing negative results may not only save resources for the participants but also help prevent previously observed adverse events from recurring, especially in the research of drug discovery. Participants offer informed consent for research under the circumstances of benefits outweighing harm to facilitate scientific development. These participants expose themselves to risk and trust the research team. It is a moral obligation for researchers to report and disseminate the results irrespective of the outcomes.

\section{Challenges of publishing negative results}

Many challenges discourage different contributors from publishing manuscripts with negative results. There is no doubt that many journal editors prefer to publish studies with positive results which are more interesting and will attract more citations. For journals, more citations can contribute to better reputation, higher quality submissions, and more advertising revenues. On the contrary, editors might take the opposite view since procedures or treatments proven ineffective by negative studies could lead to subsequent omission of their use.

From the perspective of researchers, they are also more likely to choose not to submit studies with negative results. However, the major reason for this is lack of time and priority rather than fear of rejection by the journals. ${ }^{13}$ They may turn to investigate other novel and promising research projects instead of writing up the results of a negative trial. Among the fields of hot research topics, there are many more options for them to study. With negative studies published, they may be reluctant to admit that they had selected the wrong hypothesis.

Other stakeholders, such as pharmaceutical companies or sponsors may also prefer not to disseminate negative findings. Clinical trials sponsored by industry are less likely to get published compared to studies initiated by the academia. ${ }^{18}$ For clinicians, it is relatively difficult to incorporate negative study findings into clinical practice owing to improper dissemination of such study findings; poorly designed decision tools for clinical use; and confusion caused by inconsistent study results, scepticism of new data, and information overload. ${ }^{19}$

\section{Way forward-and Journal policy}

The problem of unethical publication bias has led many academic organisations, including the International Committee of Medical Journal Editors (ICMJE) ${ }^{20}$ the World Health Organization, ${ }^{21}$ and the Committee on Publication Ethics (COPE) $)^{22}$ to implement relevant recommendations and guidelines that recommend journals require publishing the registration number of clinical trials and support disseminating the findings of previously unreported clinical trials. ${ }^{20,21}$ This is also supported by the Consolidated Standards of Reporting Trials Statement (CONSORT) ${ }^{23}$ guidelines and the Declaration of Helsinki. ${ }^{24}$ Some journals offer publication of trial protocols in advance of completion of the study, with an undertaking to publish the results irrespective of whether they are positive or negative.

Researchers have now formed an All Trials campaign to support reporting unpublished clinical studies owing to the observed irreproducibility of many published studies. The campaign endorses publication of negative findings to gather all data on the evaluation of interventions. ${ }^{25}$ Reviewers and editors should not bury studies investigating important research questions that fail to illustrate a treatment effect. Care should also be taken to differentiate true negative findings from lowquality studies, to ensure the results are not caused by chance. Some journals publish mainly negative findings, such as the Journal of Pharmaceutical Negative Results; however, others have already ceased publication, such as the Journal of Negative Results in BioMedicine. It is uncertain if such journals might produce bias, as publication of studies with negative findings is preferred. Standards for publishing are the study quality and statistical power regardless of the results, and appropriate study design in nonsuperiority and equivalence trials. The findings from well-conducted research can be trusted irrespective of proving or rejecting the null hypothesis.

We wish to emphasise that the Hong Kong Medical Journal is committed to publishing highquality reports of research relevant to the journal's scope for clinical practice, including those with negative results. ${ }^{26} \mathrm{~A}$ well-performed negative study is a positive contribution to science and clinical 
practice, and can contribute to the judicious use of healthcare resources. The relevance of research questions and the quality of the methodology are the important aspects we wish to evaluate. We suggest that research outcomes should be reported for articles irrespective of their statistical significance and they should comply with the reporting guidelines from relevant organisations or academic groups. It is also advised to report effect size and confidence intervals for all clinical outcomes. To conclude, researchers, reviewers, editors, readers, and sponsors need to be aware of the importance of negative findings and promote disseminating negative and positive results alike.

\section{Author contributions}

MCS Wong and JH Huang contributed to the drafting of the article; D Weller and R Jones reviewed and revised the article. All authors approved the final version for publication.

\section{Conflicts of interest}

The authors have declared no conflicts of interest.

\section{Funding/support}

This editorial received no specific grant from any funding agency in the public, commercial, or not-for-profit sectors.

\section{References}

1. Pasternak JJ, Williamson EE. Clinical pharmacology, uses, and adverse reactions of iodinated contrast agents: a primer for the non-radiologist. Mayo Clinic 2012;87:390-402.

2. Hinson JS, Al Jalbout N, Ehmann MR, Klein EY. Acute kidney injury following contrast media administration in the septic patient: A retrospective propensity-matched analysis. J Crit Care 2019;51:111-6.

3. Poston JT, Koyner JL. Sepsis associated acute kidney injury. BMJ 2019;364:k4891.

4. Lam SM, Lau AC, Lam RP, Yan WW. Clinical management of sepsis. Hong Kong Med J 2017;23:296-305.

5. Hsu YC, Su HY, Sun CK, Liang CY, Chen TB, Hsu CW. Risk of post-contrast acute kidney injury in emergency department patients with sepsis. Hong Kong Med J 2019;25:429-37.

6. Lo W, Fung GP, Cheung PC. Factors associated with multidisciplinary case conference outcomes in children admitted to a regional hospital in Hong Kong with suspected child abuse: a retrospective case series with internal comparison. Hong Kong Med J 2017;23:454-61.

7. Cheung TK, Cheng TC, Wong LY. Willingness for deceased organ donation under different legislative systems in Hong Kong: population-based cross-sectional survey. Hong Kong Med J 2018;24:119-27.

8. Chow JF, Yeung WS, Lee VC, Lau EY, Ho PC, Ng EH. Preimplantation genetic diagnosis and screening by array comparative genomic hybridisation: experience of more than
100 cases in a single centre. Hong Kong Med J 2017;23:12933.

9. Cheung MY, Ho AW, Wong SH. Post-fracture care gap: a retrospective population-based analysis of Hong Kong from 2009 to 2012. Hong Kong Med J 2018;24:579-83.

10. Duyx B, Urlings MJ, Swaen GM, Bouter LM, Zeegers MP. Scientific citations favor positive results: a systematic review and meta-analysis. J Clin Epidemiol 2017;88:92-101.

11. Dickersin K, Chan S, Chalmers TC, Sacks HS, Smith H Jr. Publication bias and clinical trials. Control Clin Trials 1987;8:343-53.

12. Fanelli D. Negative results are disappearing from most disciplines and countries. Scientometrics 2012;90:891-904.

13. Song F, Parekh S, Hooper L, et al. Dissemination and publication of research findings: an updated review of related biases. Health Technol Assess 2010;14:iii,ix-xi,1-193.

14. Sterne JA, Sutton AJ, Ioannidis JP, et al. Recommendations for examining and interpreting funnel plot asymmetry in metaanalyses of randomised controlled trials. BMJ 2011;343:d4002.

15. Connor JT. Positive reasons for publishing negative findings. Am J Gastroenterol 2008;103:2181-3.

16. Kerr NL. HARKing: hypothesizing after the results are known. Pers Soc Psychol Rev 1998;2:196-217.

17. Rahman MS, Yoshida N, Tsuboi $\mathrm{H}$, et al. The health consequences of falsified medicines-A study of the published literature. Trop Med Int Health 2018;23:1294-303.

18. Stefaniak JD, Lam TC, Sim NE, Al-Shahi Salman R, Breen DP. Discontinuation and non-publication of neurodegenerative disease trials: a cross-sectional analysis. Eur J Neurol 2017;24:1071-6.

19. Mitka M. Clinicians remain reluctant to allow negative findings to influence practice. JAMA 2012;308:1305-6.

20. International Committee of Medical Journal Editors. Recommendations for the Conduct, Reporting, Editing, and Publication of Scholarly Work in Medical Journals. Available from: http://www.icmje.org/recommendations/. Accessed 17 Nov 2019.

21. World Health Organization. Statement on Public Disclosure of Clinical Trial Results. Available from: http://www.who.int/ ictrp/results/reporting/en/. Accessed 17 Nov 2019.

22. Committee on Publication Ethics. Code of conduct and best practice guidelines for journal editors. Available from: https:// publicationethics.org/files/Code_of_conduct_for_journal_ editors_Mar11.pdf. Accessed 17 Nov 2019.

23. Schulz KF, Altman DG, Moher D; CONSORT Group. CONSORT 2010 statement: updated guidelines for reporting parallel group randomised trials. BMJ 2010;340:c332.

24. World Medical Association. World Medical Association Declaration of Helsinki: ethical principles for medical research involving human subjects. JAMA 2013;310:2191-4.

25. AllTrials Campaign. All trials registered. All results reported. Available from: http://www.alltrials.net/wp-content/ uploads/2013/09/What-does-all-trials-registered-andreported-mean.pdf. Accessed 17 Nov 2019.

26. Wong MC. Exerting an impact on clinical practiceupholding quality, visibility, and timeliness of publications. Hong Kong Med J 2017;23:4-5. 